\title{
Diacronie
}

Studi di Storia Contemporanea

$\mathrm{N}^{\circ} 21,1 \mid 2015$

Le città di Babele

\section{Michel Foucault, Mal fare, dir vero. Funzione della confessione nella giustizia. Corso di Lovanio (1981)}

\section{Guglielmo Sano}

\section{Q OpenEdition}

Edizione digitale

URL: http://journals.openedition.org/diacronie/1937

DOI: 10.4000/diacronie.1937

ISSN: 2038-0925

Editore

Association culturelle Diacronie

Notizia bibliografica digitale

Guglielmo Sano, « Michel Foucault, Mal fare, dir vero. Funzione della confessione nella giustizia. Corso di Lovanio (1981) », Diacronie [Online], № 21, 1 | 2015, documento 13, Messo online il 01 mars 2015, consultato il 22 septembre 2020. URL : http://journals.openedition.org/diacronie/1937 ; DOI : https:// doi.org/10.4000/diacronie.1937 


\section{RECENSIONE:}

\section{Michel FOUCAULT, Mal fare, dir vero.} Funzione della confessione nella giustizia. Corso di Lovanio (1981), Torino, Einaudi , 2013, XVI+352 pp.

a cura di Guglielmo SANO *

Il problema della soggettività e, strettamente connesso ad esso, quello della verità e della loro relazione sono due questioni che si dipanano lungo tutta la storia occidentale, come dimostra Michel Foucault nel corso che tenne a Lovanio nel 1981, recentemente edito in edizione italiana da Einaudi.

Parte degli studi foucaultiani sulla disciplina e il condizionamento sociale e politico, il corso e il seminario tenuti nel 1981 sulla "difesa sociale", rispondono all'invito della Scuola di Criminologia della Facoltà di Diritto dell'Università Cattolica di Lovanio. Invito accolto da Foucault, professore del Collège de France, grazie alla stima che nutre nei confronti di Francoise Tulkens, una giovane professoressa della Facoltà di diritto e futura presidente della Corte Europea per i diritti dell'uomo.

Tulkens, insieme ad altri penalisti attivi a Lovanio - Michel Van de Kerchove e Foulek Ringheleim - anch'essi in contatto con Foucault, si era messa in evidenza grazie a un rapporto, del 1979, sul processo di revisione del codice penale Belga del 1867 (poi corredato di leggi complementari sul vagabondaggio e la mendicità nel 1891 e sugli anormali e i delinquenti abituali nel 1930) ritenuto "espressione del diritto penale classico". Nel rapporto, molto critico, della Tulkens veniva evidenziato che il Codice Penale Belga criminalizzava alcuni "tipi” di individui per internarli, in base a una nozione di "pericolosità" influenzata dai promotori della difesa sociale e dalle nascenti "scienze criminali". Il rapporto, con la sua radicalità, si pone in chiara sintonia con l'indagine foucaultiana come dimostrano il seminario e il corso tenuti da Foucault, i 
quali contribuiranno al dibattito sulla riforma penale belga, minando la nozione di "difesa sociale" dalle fondamenta.

Il corso, intitolato Mal Fare, dir vero, è composto da una conferenza inaugurale e da sei lezioni, il cui intento è quello di delineare una storia della confessione come forma di legame e di rapporto tra "veridizione" e "giurisdizione":

non esiste confessione in senso stretto se non all'interno di una relazione di potere alla quale la confessione dà modo di esercitarsi su colui che confessa. Le cose sono evidenti quando le relazioni di potere sono definite istituzionalmente: come nel caso della confessione giudiziaria, o della confessione nella Chiesa Cattolica ${ }^{1}$.

Foucault, quindi, si interroga sulla genesi e sulla funzione del dire la verità nella pratica giudiziaria, mettendo in evidenza come nella pratica moderna "dire la verità" assume la forma del "dire la verità su di sé". Questo punto viene chiarito dall'autore attraverso vari esempi di tecniche di veridizione riscontrabili nella storia occidentale: l'esame di coscienza degli stoici, la penitenza nel cristianesimo primitivo, l'esame di sé dei monaci del V secolo, la confessione sacramentale organizzata dal Concilio Laterano, la perizia psichiatrica, la psicanalisi, etc.

Nel corso all'Università Cattolica Foucault tenta di rispondere ad alcune domande pressanti tra cui, "come intendere la proliferazione dei modi di dire la verità e la variabilità dei regimi di veridizione in società come la nostra?”, "Qual è l'unità di queste forme di veridizione?”, “Quali le differenze?”:

definire nella loro pluralità i modi di veridizione, di ricercare le forme di obbligazione attraverso cui ciascuno dei modi vincola il soggetto del dir vero, di specificare le aree a cui si applicano e gli ambiti di oggetti che fanno apparire, infine le relazioni, connessioni, interferenze che sono stabilite tra di essi ${ }^{2}$.

Foucault non è nuovo all' impresa avendo già testato il suo metodo, archeologico e genealogico, su pratiche sociali come l'internamento dei folli e la carcerazione dei criminali. Interrogarsi sulla "confessione”, quindi, per Foucault rientra nell'indagine, costantemente portata avanti dal pensatore francese, che verte sulla formazione dei modi di dire la verità; sui modi di dire qualcosa che viene considerato una "verità". In questo quadro, Foucault, problematizza la sua analisi chiedendosi non a quali

\footnotetext{
${ }^{1}$ FOUCAULT, Michel, Mal fare, dir vero. Funzione della confessione nella giustizia. Corso di Lovanio (1981), Torino, Einaudi, 2013, p. 8.

${ }^{2}$ Ibidem, p. 12.
} 
condizioni un enunciato sia vero o falso, ma a quali condizioni si sia formata una modalità di dire la verità, piuttosto che un'altra. Adottata questa prospettiva si capisce come le lezioni in oggetto si occupino di studiare le modalità di apparizione, di consolidamento e trasformazione della "veridizione di sé" nella pratica giudiziaria.

In questo caso la confessione non consiste nel riconoscere una colpa o un atto davanti a un'istanza giudiziaria che chiede di riferirle quello che è successo. Il senso della confessione di Antiloco è legato alla gara che non intendeva decretare un vincitore imprevisto tra concorrenti di pari forze, ma realizzare un ordine già inscritto nelle cose, cioè la vittoria del concorrente più forte a sua volta sostenuto dagli dei. La gara è, quindi, una "liturgia della verità" e Achille ne è il suo officiante. L'irregolarità di Antiloco ha impedito alla verità di manifestarsi e la confessione è il rito attraverso cui ristabilire l'ordine. Il carattere giudiziario attiene al fatto che, Achille, attui una procedura conforme alle regole della Themis - personificazione di ordine, legge e giustizia - cioè alle regole che permettono lo svolgimento dei riti.

Foucault ritrova anche in Esiodo degli spunti di riflessione, per esempio nella scena dei Contendenti, quando il giudice registra come propria sentenza il confronto regolato tra le parti, oppure, in un altro luogo esiodeo, il giudice presta giuramento a sua volta e si riferisce a un ambito di giustizia diverso da quello della Themis. Al giudice, pertanto, non si chiede più di regolarsi a un ordine, a una giustizia inscritta nelle cose, adesso gli si chiede di essere "giusto" nella propria sentenza: la comparsa del dikaion emerge insieme alla figura di un giudice che presta giuramento e si dichiara superiore alle parti. Questa, secondo Foucault, è la prima forma di articolazione tra vero e giusto, che rimarrà sempre problematica nell'ambito della giustizia e, a partire dal XIX secolo, attribuirà alla confessione il compito di unire e far funzionare il principio di verità e il principio di giustizia.

Successivamente, Foucault passa ad analizzare la figura di Edipo nella tragedia Edipo Re di Sofocle. Piuttosto che interessarsi al fatto commesso da Edipo, tuttavia, Foucault riflette sulle modalità con le quali è Edipo stesso a far emergere la verità. Nella tragedia si possono distinguere tre veridizioni successive. La prima, quella di dei e profeti, si presenta attraverso le figure di Creonte - inviato da Edipo ad interrogare il dio Apollo - e l'indovino Tiresia. La seconda, invece, vede come protagonisti Edipo e Giocasta, i quali confrontando i propri ricordi disvelano la verità. La "verità", ormai emersa come "verità giudiziaria", nondimeno, viene accettata solamente dopo la terza opera di veridizione, quando il messaggero di Corinto e il pastore del Citerone incolpano Edipo. Dal momento che questi due personaggi parlano di quello che hanno visto e hanno fatto, la loro procedura di veridizione e la loro "testimonianza di 
confessione" non può essere messa in discussione né dalle parole divinatorie e profetiche, né dalle tecniche deduttive del Re. Lo stesso Edipo ammetterà la colpa dirà "io" - solo una volta che il coro (l'Altro) avrà ascoltato e accettato la verità della "testimonianza di confessione" dei servi. Nell'Edipo Re, quindi, Foucault vede la rappresentazione drammatica della nuova pratica giudiziaria ateniese che faceva della

Il monachesimo, nell'analisi foucaultiana, integra le precedenti pratiche penitenziali e le pratiche filosofiche dell'antichità pagana, l'accesso alla verità è regolato dalla mortificazione di sé e dall'obbedienza a un direttore spirituale, un rapporto che deve essere continuo e permanente, restando comunque molto diverso dal rapporto discepolo-maestro di Seneca che si interrompeva una volta che il discepolo acquisiva la saggezza. Per arrivare alla totale obbedienza, tuttavia, bisognava "parlare”. Bisognava dire al proprio direttore spirituale tutto quello che succedeva dentro di sé, ogni movimento del pensiero, perché, proprio il pensiero, agitandosi può distogliere il monaco dalla contemplazione di Dio. Per questo deve essere costantemente controllata la qualità e l'origine del pensiero, così da poterlo controllare. In altre parole, non ci può essere conoscenza di sé - il monaco non può sapere quali pensieri si siano ancorati nella sua coscienza - se non attraverso atti verbali, continuamente ripetuti e riproposti; sebbene la verbalizzazione di sé fatta all'altro si trasformi nel medesimo tempo nella pratica d'accesso alla rinuncia a se stessi.

confessione una pratica fondamentale del sistema giudiziario ma, la tragedia edipica, consente anche di rintracciare la genealogia di un'ermeneutica di sé, attiva nelle pratiche della confessione, che la macchina giudiziaria contemporanea esige.

L'obbligo di "dire la verità" su di sé fa quindi la sua comparsa nella storia occidentale, con la pratica monastica, come strumento di mortificazione di sé e di conoscenza di sé. Ritroviamo l'ermeneutica di sé - la volontà di conoscersi, di accedere alla verità dei propri pensieri e della propria soggettività, di scoprire la propria natura, di conoscere i propri sentimenti, di scoprire la verità del proprio desiderio, di ritrovare il senso delle proprie azioni, e di valutarlo all'interno di un atto verbale o scritto - in molte espressioni moderne e contemporanee. Innanzitutto nelle pratiche giudiziarie e psichiatriche, ma anche in filosofia e in letteratura, nella religione come nella psicologia come dimostrano le riflessioni di Cartesio, Schopenhauer e Freud sul pensiero e sulla possibilità che ha di ingannarsi.

Foucault, quindi, traccia una storia della verità e della soggettività nella cultura occidentale localizzando nella confessione il centro e il motore di questa problematizzazione del Soggetto. In seguito, partendo da questa considerazione, 
Foucault mette in evidenza come nell'istituzione penale moderna a essere giudicati non sono più gli atti ma ciò che la soggettività dice di sé sulla scena giudiziaria; a essere importante nella confessione non sono più i fatti ma le immagini, le rappresentazioni, le volontà, i desideri e i pensieri: tutto ciò che i padri latini chiamavano cogitationes. Dire la verità su di sé diventerà l'oggetto di una codificazione giuridica all'interno dell'istituzione ecclesiastica che, nel XII secolo, porterà alla sacramentalizzazione della confessione: penitente e confessore si muoveranno in base a un vero e proprio codice di colpe e penitenze, che influenzerà molto il diritto post-medievale.

Confessare, pertanto, sin dai codici del XVII secolo, equivarrà a riconoscere il sistema penale da cui si viene condannati, il patto sociale che si è infranto con il delitto; non si cercano prove ma la relazione tra la soggettività criminale e il crimine. Chi confessa restaura il patto sociale e si impegna, attraverso la punizione, sulla via della correzione.

Questo meccanismo di auto-veridizione non solo rafforza l'adesione del cittadino al patto sociale ma anche l'ideologia del sistema politico:

La confessione legando il soggetto a quanto afferma, lo qualifica in modo diverso rispetto a ciò che dice: criminale ma forse suscettibile di pentirsi; innamorato, ma dichiarato; malato, ma già sufficientemente cosciente e distaccato dalla propria malattia perché possa lavorare in prima persona alla propria guarigione. Diciamo, in poche parole, e per riassumere tutto quello che abbiamo detto, che la confessione è un atto verbale attraverso cui il soggetto fa un'affermazione su ciò che egli è, si lega a questa verità. Si colloca in un rapporto di dipendenza nei confronti di altri, e modifica nello stesso tempo il rapporto che ha con se stesso3.

Ma anche il meccanismo di auto-veridizione, così espresso, verrà sorpassato all'incirca nella prima metà dell'Ottocento. Non cercando più prove, ma la relazione tra soggetto criminale e crimine, al giudice sfuggivano molte cose sui delitti senza ragione e senza confessione. Quelli senza ragione non potevano essere spiegati attraverso la ragione, la follia, la passione o l'interesse, quelli senza confessione, invece, vedevano i colpevoli, che avevano riconosciuto e ammesso i fatti, incapaci di spiegarne qualcosa in proposito. A questo punto toccherà all'esame psichiatrico colmare le lacune dell'autoveridizione del colpevole e pronunciarsi su tali crimini. L'atto individuale verrà spiegato sempre più in termini di relazioni di senso e di relazioni inconsce.

Foucault, in ultima analisi, nota che nello stesso periodo compare nel diritto civile una dottrina della responsabilità senza colpa, per cui la responsabilità non attiene né

\footnotetext{
${ }^{3}$ Ibidem, p. 9 .
} 
alla volontà né alla colpa del soggetto, ma al rischio che fa correre a un terzo. Su questa base, dice Foucault, si instaureranno i tentativi di fondare il sistema penale sulla nozione di pericolosità, ovvero, colmando le lacune della confessione nel sistema di difesa sociale con la "responsabilità senza colpa" e con la nozione di "rischio" collettivo. 


\section{* L'autore}

Guglielmo Sano si è laureato in "Filosofia, conoscenza e comunicazione" presso l'Università di Palermo, con un lavoro sulla filosofia del linguaggio di Michel Foucault. Attualmente frequenta il corso di Laurea Magistrale in "Scienze Filosofiche" presso l’Università di Bologna.

URL: < http://www.studistorici.com/progett/autori/\#Sano >

\section{Per citare questo articolo:}

SANO, Guglielmo, «Recensione: Anna TONELLI, Gli irregolari. Amori comunisti al tempo della Guerra fredda, RomaBari, Laterza, 2014, 192 pp.», Diacronie. Studi di Storia Contemporanea : Le città di Babele, 29/3/2015, URL:< http://www.studistorici.com/2015/03/29/sano_numero_21/>

Diacronie Studi di Storia Contemporanea $\}$ www.diacronie.it

Risorsa digitale indipendente a carattere storiografico. Uscita trimestrale.

redazione.diacronie@hotmail.it

Comitato di redazione: Jacopo Bassi - Luca Bufarale - Elisa Grandi - Deborah Paci - Fausto Pietrancosta - Matteo Tomasoni - Luca Zuccolo 